Proc. XIX International School of Semiconducting Compounds, Jaszowiec 1990

\title{
NEUTRON TRANSMUTATION DOPED GaP: OPTICALLY DETECTED MAGNETIC RESONANCE STUDIES*
}

\author{
B.J. Heijmink Liesert ${ }^{a}$ ), M. Godlewski ${ }^{b}$ ), T. Gregorkiewicz ${ }^{a}$ ), \\ C.A.J. AMmerlaAN ${ }^{a)}$ AND E. Goldys ${ }^{c}$ ) \\ a) Natuurkundig Laboratorium, Universiteit van Amsterdam, Valckenierstraat 65, \\ NL-1018 XE Amsterdam, The Netherlands \\ b)Institute of Physics, Polish Academy of Sciences, 02-668 Warszawa, Al. \\ Lotników 32/46, Poland \\ c) Institute of Experimental Physics, Warsaw University, Hoża 69, 00-681 Warszawa, \\ Poland
}

(Received August 8, 1990)

The first direct proof for successful neutron transmutation doping (NTD) of $\mathrm{GaP}$ is presented on the basis of optically detected magnetic resonance (ODMR). GaP:S samples grown by the liquid encapsulated Czochralski method were irradiated with thermal neutrons and subsequently annealed at $800^{\circ} \mathrm{C}$. In the ODMR experiments the transmuted Ge substitutional on Ga sites was detected. The NTD process was also found to create deep acceptors, the nature of which will be tentatively discussed.

PACS numbers: $61.80 . \mathrm{Hg}, 76.70 . \mathrm{Hb}$

\section{Introduction}

Neutron transmutation doping (NTD) is a well established method for $n$-type doping of Si. The quality of high-power electronic devices was highly improved due to doping homogeneity and low defect concentration of the NTD Si. Much less attention was paid to transmutation doping of III-V compounds. Till very recently

'This work received financial support from the Foundation for Fundamental Research on Matter (FOM). 
no definite proof existed of successful NTD of GaP in which Ge donors are expected to be formed. Huber and co-workers [1], who applied a rather small neutron fluence, could only indirectly conclude on NTD of GaP.
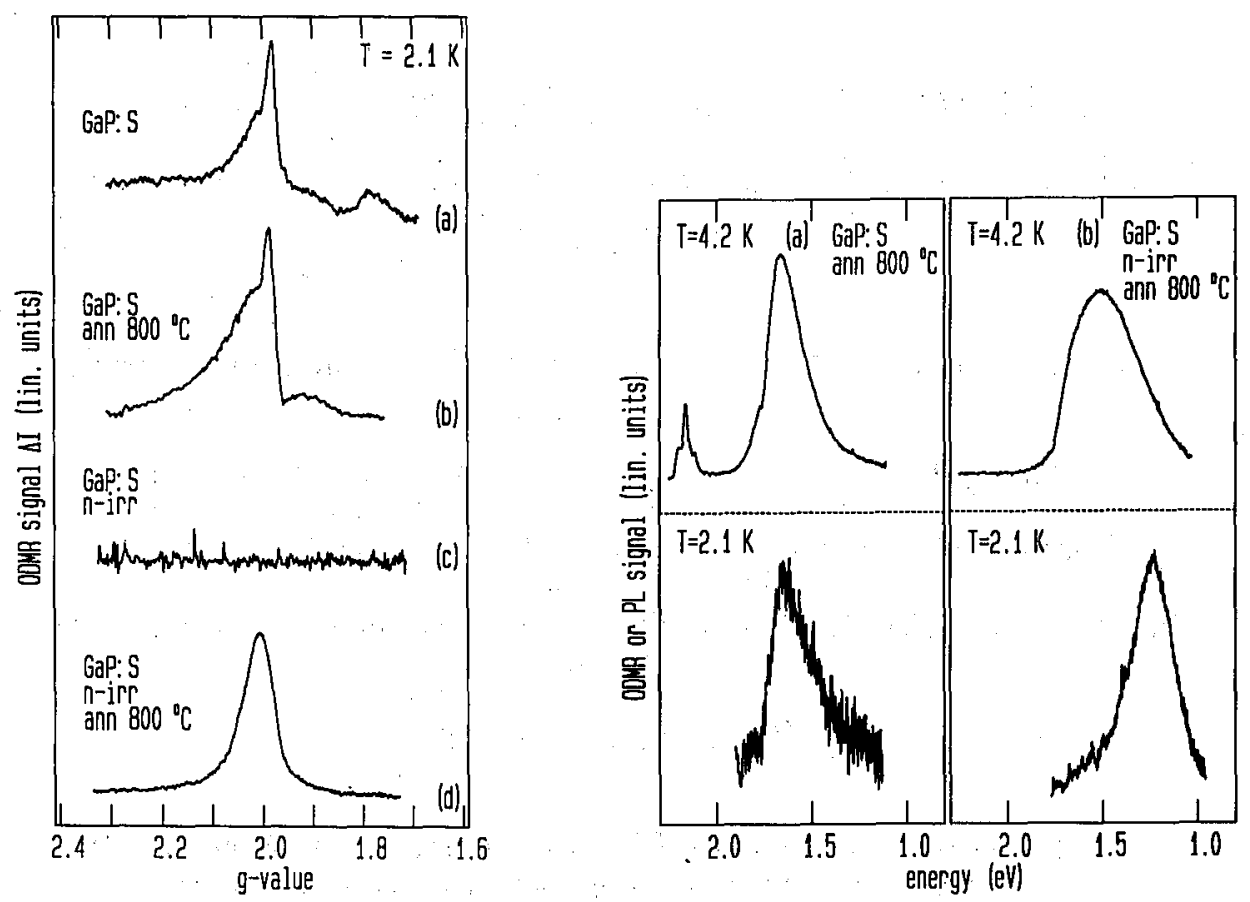

Fig. 1. ODMR spectra measured at $2.1 \mathrm{~K}$ of (a) the starting material LEC GaP:S, (b) annealed $\left(800^{\circ} \mathrm{C}, 1 \mathrm{~h}\right),(\mathrm{c})$ as-irradiated and (d) irradiated and subsequently annealed $\left(800^{\circ} \mathrm{C}, 1 \mathrm{~h}\right)$. Changes of the total infrared emission $(\Delta \mathrm{I})$ under $514.5 \mathrm{~nm} \mathrm{Ar}{ }^{+}$-excitation are plotted against $g$-value.

Fig. 2. Photoluminescence (top) and spectral dependence of the donor ODMR signals (bottom) for (a) the starting material LEC GaP:S annealed $\left(800^{\circ} \mathrm{C}, 1 \mathrm{~h}\right)$ and (b) irradiated and subseqently annealed $\left(800^{\circ}, 1 \mathrm{~h}\right)$. Spectra are measured through a $1.5 \mathrm{~m}$ monochromator with a Ge-detector using a $514.5 \mathrm{~nm} \mathrm{Ar}^{+}$-line for excitation.

In the optically detected magnetic resonance (ODMR) studies presented here the starting material was commercially available $n$-type GaP:S grown by the liquid encapsulated Czochralski (LEC) method. The doping level prior to irradiation was $7 \times 10^{17} \mathrm{~cm}^{-3}$. Neutron irradiation was performed at Świerk Nuclear Research Center in Poland with the ratio of thermal to fast neutrons being 1000:1. The thermal neutron fluence was $1.4 \times 10^{19} \mathrm{~cm}^{-2}$. After irradiation the samples were vacuum annealed for $1 \mathrm{~h}$ at $800^{\circ} \mathrm{C}$. Hall effect measurements [2] showed $n$-type sample conductivity with the thermal activation energy of $200 \pm 10 \mathrm{meV}$. This agrees well with the value of $204 \mathrm{meV}$ reported for $\mathrm{GaP}$ conventionally doped 
with Ge and is thus indicative of successful NTD.

In this paper we will show that after irradiation and annealing the transmuted $\mathrm{Ge}\left({ }^{69(71)} \mathrm{Ga}+\mathrm{n} \rightarrow{ }^{70(72)} \mathrm{Ga}\left(\beta^{-}\right) \rightarrow{ }^{70(72)} \mathrm{Ge}\right)$ enters the $\mathrm{Ga}$ site forming $\mathrm{Ge}_{\mathrm{Ga}}$ donor states. Secondly, the observation of Huber et al. [1] will be confirmed that NTD introduces also some acceptor states which are not annealed out even at $800^{\circ} \mathrm{C}$. The nature of these deep Ge related acceptors will be discussed.

\section{Results}

The ODMR experiments were carried out at $2.1 \mathrm{~K}$ at $35 \mathrm{GHz}$, using a split-coil superconducting magnet in the Faraday configuration. Samples were mount in a cylindrical $\mathrm{TE}_{011}$ cavity with slits for optical access. Luminescence (PL) was excited with a $514.5 \mathrm{~nm} \mathrm{Ar}^{+}$-laser line. It was dispersed by a $1.5 \mathrm{~m}$ high-resolution monochromator and detected with a Ge-detector using standard lock-in techniques. More experimental details may be found elsewhere [3].

The ODMR spectra, measured via monitoring the change of the total infrared PL in resonance, are shown in Fig. 1. The spectral dependence of the ODMR signals was investigated by setting the magnetic field to resonance and scanning through the luminescence. Relevant experimental data are plotted in Fig. 2.

\section{Discussion and conclusions}

The characteristic sulphur donor resonance signal with an isotropic value of $g=1.99 \pm 0.01$ is observed for the starting material and also following $1 \mathrm{~h} 800^{\circ} \mathrm{C}$ annealing - Fig. 1(a,b). The as-irradiated material was non-transparent due to a high concentration of radiation defects caused by $\beta$ and $\gamma$ recoil during transmutation. Hence, no ODMR spectrum could be observed - Fig. 1(c). After neutron irradiation and subsequent $1 \mathrm{~h} 800^{\circ} \mathrm{C}$ annealing a new isotropic resonance signal with $g=2.00 \pm 0.01$ was found - Fig. 1(d). The identical resonance spectrum was previously observed for conventionally doped LEC GaP:Ge, both in ESR [4] and ODMR [5]. Consequently, we identify this signal with the substitutional $\mathrm{Ge}$ donor center. This donor signal is an enhancement of the $1.25 \mathrm{eV}$ emission, see Fig. 2(b), proving the donor-acceptor pair (DAP) nature of this emission.

We wish to point out that the majority of transmuted Ge atoms is active as donors on $\mathrm{Ga}$ sites. If $\mathrm{Ge}_{\mathrm{P}}$ acceptors $\left(E_{\mathrm{A}}=E_{\mathrm{V}}+258 \mathrm{meV}\right.$ [6]) become active in $\mathrm{Ge}_{\mathrm{Ge}}-\mathrm{Ge}_{\mathbf{P}}$ DAP processes, near-edge emission should be detected. This is not confirmed by our studies, supporting the conclusions of Huber et al. [1] for NTD $\mathrm{GaP}$ and Satoh et al. [7] for NTD GaAs that major part of the transmuted Ge enters the Ga site.

The distinct shift $(\approx 200-250 \mathrm{meV}$ ) of the two PL bands from $1.5 \mathrm{eV}$ and $1.7 \mathrm{eV}$ (for LEC GaP:S) to $1.25 \mathrm{eV}$ and $1.52 \mathrm{eV}$ (for NTD GaP), see Fig. 2(a) and (b), is two times larger than the one expected to result from the change of donor ionisation energy only $\left(E_{\mathrm{D}}=107 \mathrm{meV}\right.$ for $S_{\mathrm{P}}, E_{\mathrm{D}}=204 \mathrm{meV}$ for $\mathrm{Ge}_{\mathrm{Ga}}$ [6]). This confirms previous observations $[5,8,9]$ that the acceptor energy level depends on the donor impurity introduced into the sample. From PL studies of GaP doped 
with chalcogenides (S, Se, Te) Dishman et al. [8] proposed that the actual donor species present in the material also participates in the formation of an acceptor. The deep acceptor active in the $1.5 \mathrm{eV}$ DAP transition was tentatively identified as a complex incorporating a gallium vacancy $\left(V_{\mathrm{Ga}_{\mathrm{a}}}\right)$ and two donors [8]. Extension of the Dishman model to our data would require that $V_{\mathrm{Ga}}-S_{\mathrm{P}}$ acceptors present in the starting material are replaced by $V_{\mathrm{Ga}}-\mathrm{Ge}_{\mathrm{Ga}}$ defects. This would further imply that part of the gallium vacancies, being the dominant structural defects formed by neutron irradiation $[10,11]$, is stabilised by the formation of $V_{\mathrm{Ga}}-\mathrm{Ge}_{\mathrm{Ga}}$ complex centers.

Summarising, the observation of the $\mathrm{Ge}_{\mathrm{Ga}}$ related donor ODMR signal directly confirms successful transmutation proving its feasibility for GaP. Additionally, NTD was also found to create deep acceptors, tentatively identified as $V_{\mathrm{Ga}}-\mathrm{Ge}_{\mathrm{Ga}}$ complexes.

\section{References}

[1] A. Huber, F. Kuchar, J. Casta, J. Appl. Phys. 55, 353 (1984).

[2] J. Barczyńska, E. Goldys, MRS Fall Meeting, Boston 1989 (in press).

[3] B.J. Heijmink Liesert, M. Godlewski, T. Gregorkiewicz, C.A.J. Ammerlaan, to be published.

[4] F. Mehran, T.N. Morgan, R.S. Title, S.E. Blum, Solid Slate Commun. 11, 661 (1972).

[5] M. Godlewski, B. Monemar, J. Appl. Phys. 64, 200 (1988).

[6] Landolt-Börnstein, Numerical Data and Functional Relationships in Science and Technology, Group III, Vol. 22b. eds. O. Madelung, M. Schulz, Springer-Verlag, Berlin, Heidelberg 1989, p. 511.

[7] M. Satoh, K. Kuriyama, Y. Makita, J. Appl. Phys. 65, 2248 (1989).

[8] J.M. Dishman, D.F. Daly, W.P. Knox, J. Appl. Phys. 43, 4693 (1972).

[9] O.O. Awadelkarim, M. Godlewski, B. Monemar, in Proc. 15th Internat. Conf. Defects in Semiconductors, Budapest 1988, in series Mater. Science Forum 38-41, ed. G. Ferenczi, Trans Tech Publications, Switzerland 1989, p. 821.

[10] T. Kawakubo, M. Okada, Phys. Status Solidi B 153, K93 (1989).

[11] G. Dlubek, C. Ascheron, R. Krause, H. Erhard, D. Klimm, Phys. Status Solidi A 106, 81 (1988). 